\title{
Influencia de la interacción dinámica suelo-estructura en el mecanismo de colapso y nivel de desempeño de edificaciones de hormigón armado
}

\section{Influence of Dynamic Soil-Structure Interaction on the Failure Mechanism and Performance Level of Reinforced Concrete Buildings}

\author{
Zenayda Corratgé Yzaguirrea, Janet O. Martínez Cid ${ }^{\mathrm{b}}$, Willian Cobelo Cristiá ${ }^{\mathrm{b}}$ \\ ${ }^{a}$ Msc. Ing. Civil. Universidad Tecnológica de la Habana José Antonio Echeverría. (La Habana, Cuba) \\ ${ }^{b}$ Dr. Ing. Civil. Universidad Tecnológica de la Habana José Antonio Echeverría. (La Habana, Cuba)
}

Recibido el 11 de agosto de 2020; aceptado el 9 de abril de 2021

\section{RESUMEN}

En el presente artículo se analiza la influencia de la consideración de la interacción dinámica suelo- estructura (IDSE) en el comportamiento estructural de una edificación de diez niveles de hormigón armado con tipología mixta sometida a carga sísmica. Para ello se implementó el método estático no lineal push-over mediante el software ETABS V17. La IDSE es incorporada a través de los modelos de Pais \& Kausel y Sargsian, considerándose las características de dos tipos de suelos: rígido y arcilla blanda contemplados en la NC 46:2017 [1]. Como principales resultados se observa, al incluir la IDSE, un aumento en los valores de desplazamiento en el tope de la edificación y de derivas por piso y una disminución de los valores de cortante basal para los cuales se alcanzan la fluencia global de la estructura y el mecanismo de colapso respectivamente. Adicionalmente, el nivel de desempeño de esta se aleja de lo recomendado por las normas.

2022 Asociación Española de Ingeniería Estructural (ACHE). Publicado por Cinter Divulgación Técnica S.L. Licencia de uso Creative Commons (CC BY-NC-ND 3.0).

PALABRAS CLAVE: Interacción dinámica suelo-estructura; análisis sísmico; push-over; nivel de desempeño; rótulas plásticas.

\section{ABSTRACT}

This paper deals with the influence of considering dynamic soil-structure interaction (DSSI) on the structural behavior of a ten-story reinforced concrete mixed typology building under seismic load. In order to do so, the non-linear static push-over method was implemented using the ETABS V17 software. The DSSI was included through the Pais \& Kausel and Sargsian models, considering the characteristics of two types of soils: rigid and soft clay according to the NC 46:2017 [1]. As main results, when including the DSSI, an increase in the values of top displacement and drifts and a decrease in the values of basal shear for which the global fluency of the structure and its failure mechanism are reached, is observed. Additionally, the performance level of the structure is far from what is recommended by the codes. 2022 Asociación Española de Ingeniería Estructural (ACHE). Published by Cinter Divulgación Técnica S.L. Creative Commons License (CC BYNC-ND 3.0).

KEYWORDS: Dynamic soil-structure interaction; seismic analysis; push-over; performance level; plastic hinges.

1.

\section{INTRODUCCIÓN}

Los efectos de los sismos en las edificaciones dependen de varios factores, entre los que se encuentran las características del propio sismo, la geometría y tipología de la edificación y el suelo sobre el cual se apoya la misma. Regularmente, en el

* Persona de contacto / Corresponding author:

Correo-e / email: zenaydacy2015@gmail.com (Zenayda Corratgé Yzaguirre) análisis y diseño de edificios ubicados en zonas de alto riesgo sísmico, se consideran empotradas las bases de las columnas del primer nivel. Esta consideración implica la asunción del suelo como infinitamente rígido, obviando la realidad del fenómeno. La respuesta estructural de una edificación sometida a cargas sísmicas se encuentra influenciada por la interacción entre el 
suelo, la estructura de cimentación y la superestructura, conocida como interacción dinámica suelo-estructura (IDSE) [2].

La consideración de la IDSE en estructuras sometidas a cargas sísmicas, puede traer consigo una disminución de las solicitaciones en la edificación, debido a la capacidad que tiene el suelo para absorber parte de la energía liberada por el sismo, evitando que esta llegue a la superestructura [3]. También pueden tener lugar incrementos en el desplazamiento en el tope de la edificación y de las derivas por piso, lo cual responde a que, al no considerar un empotramiento perfecto en la base, los desplazamientos comenzarían desde esta.

La concepción de una configuración en planta y elevación adecuadas, así como un diseño estructural que permita la disipación de energía sin que tengan lugar daños significativos, constituyen un aspecto importante a tener en cuenta cuando se trata de edificios sometidos a cargas sísmicas. Con este objetivo se han establecido, por parte de distintas normativas y códigos como [4], [5], [6], [7], [8], [9], el cumplimiento del principio de columna fuerte - viga débil, garantizando la generación de un mecanismo de colapso donde las rótulas plásticas surjan, primeramente, en las vigas. Cuando se incluye la IDSE pueden ocurrir variaciones en el mecanismo de colapso al modificarse el orden y lugar de aparición de las articulaciones plásticas lo que conduce a la pérdida de la condición de "columna fuerte - viga débil ", ya que los momentos de segundo orden debidos a la carga axial que actúa sobre las columnas del primer nivel y el desplazamiento generado por el cabeceo del edificio, son suficientemente grandes como para hacer incursionar a las columnas en la zona de comportamiento inelástico. Esto hace que se reduzca la capacidad de la estructura de poder distribuir la presencia de articulaciones plásticas de una manera más equitativa, concentrándose en los niveles más bajos en el momento de la reducción de resistencia.

Los códigos establecen niveles de desempeño recomendados en función de la importancia de la obra y del tipo de sismo que sobre esta incide [10], [1]. Investigaciones [11], [12] han evidenciado que el mecanismo de colapso puede verse afectado a partir de la inclusión de la IDSE en el análisis, lo que implica que el desempeño de la edificación pueda modificarse. Estos niveles de desempeño, que describen el estado límite de daño de la edificación, al modificarse con relación a la consideración de la base como empotrada, propician la incursión en rangos en los que la seguridad y la funcionalidad de la edificación posterior al terremoto pueden verse amenazadas.

Con relación a la altura de las edificaciones a partir de la cual debe incluirse la IDSE en los modelos de análisis, los estudios no son concluyentes. Autores [13] señalan la importancia de considerar la IDSE en edificios altos cimentados sobre suelos blandos, mientras que [14] destaca que dicho fenómeno debe ser incluido tanto en suelos blandos como firmes. En [15] se analiza el comportamiento ante carga sísmica de edificios de 5, 7, 10, 12 y 20 niveles, afirmando que el único caso donde los efectos de la IDSE no resultaron significativos fue en la edificación de 20 niveles, aspecto que se asocia con la relación entre el periodo del suelo y el de la estructura.

Los métodos para la representación de la IDSE en la modelación se dividen, de manera general, en dos grupos: los métodos directos y los métodos de subestructura. Cuando se realiza un análisis directo, el suelo es representado como un continuo seguido de la cimentación y la superestructura. Entre la cimentación y el suelo se declara un elemento de interface y en los límites del suelo se declaran elementos de frontera. Estos análisis son usualmente realizados mediante la representación lineal de las propiedades del suelo a través de elementos finitos, diferencias finitas, ente otros. Estos métodos, a pesar de brindar una solución bastante exacta a los problemas de IDSE, se caracterizan por una alta complejidad desde el punto de vista computacional que representan, sobre todo cuando el sistema es geométricamente complejo o se considera la no linealidad del suelo y de los materiales estructurales.

Los métodos de subestructura, entre los cuales se encuentran las formulaciones de País \& Kausel y las de Sargsian, se caracterizan por la incorporación en los modelos de valores de rigidez equivalente en cuyo cálculo intervienen factores propios de la cimentación y del suelo sobre el cual se encuentra situada la obra. La rigidez y el amortiguamiento de la interacción suelo - cimentación son caracterizadas empleando funciones de impedancia relativamente simples o a través de una serie de resortes y amortiguadores. Dichas rigideces toman en cuenta características tales como las dimensiones de la cimentación, el módulo de cortante del suelo, el coeficiente de Poisson y los factores de corrección por profundidad de cimentación y por rigidez dinámica, brindando una adecuada aproximación al problema.

El presente trabajo tiene como objetivo identificar las modificaciones que tienen lugar en el mecanismo de colapso y en el nivel de desempeño alcanzado por una edificación de hormigón armado con tipología mixta de 10 niveles, a partir de la consideración de la IDSE en el análisis bajo la acción de carga sísmica. Se introduce la IDSE mediante las funciones de impedancia de Pais \& Kausel incluidas en [16] y las formulaciones de Sargsian incluidas en [17] y se comparan los resultados con la base empotrada. Se modifica, además, el tipo de suelo, considerando la clasificación que para estos establece la NC 46: 2017 [1].

\section{2. \\ DESCRIPCIÓN DE LA EDIFICACIÓN}

\section{Geometría}

La estructura cuenta con 10 niveles de altura, siendo el primero de $3.00 \mathrm{~m}$ y el resto de $2.70 \mathrm{~m}$, para un total de $27.30 \mathrm{~m}$. Está compuesta por pórticos y tímpanos dispuestos en 12 módulos de $7.20 \mathrm{~m}$ x $7.20 \mathrm{~m}$, no presentando irregularidades ni en planta ni en elevación En la figura 1 se muestra una planta del edificio y una vista tridimensional.

Las vigas cuentan con una sección transversal de $60 \mathrm{~cm}$ x $40 \mathrm{~cm}$ y las columnas son de sección cuadrada de $65 \mathrm{~cm} \mathrm{x}$ $65 \mathrm{~cm}$. Las losas son de $20 \mathrm{~cm}$ de espesor y los tímpanos de $25 \mathrm{~cm}$, presentando estos últimos, continuidad estructural en todos los niveles.

Para las variantes donde se incluyó la IDSE, se modeló una losa de cimentación que responde a las propiedades de los suelos utilizados (Tabla 1) acordes a la clasificación que sobre estos establece la NC 46: 2017 [1] (tabla 2). 

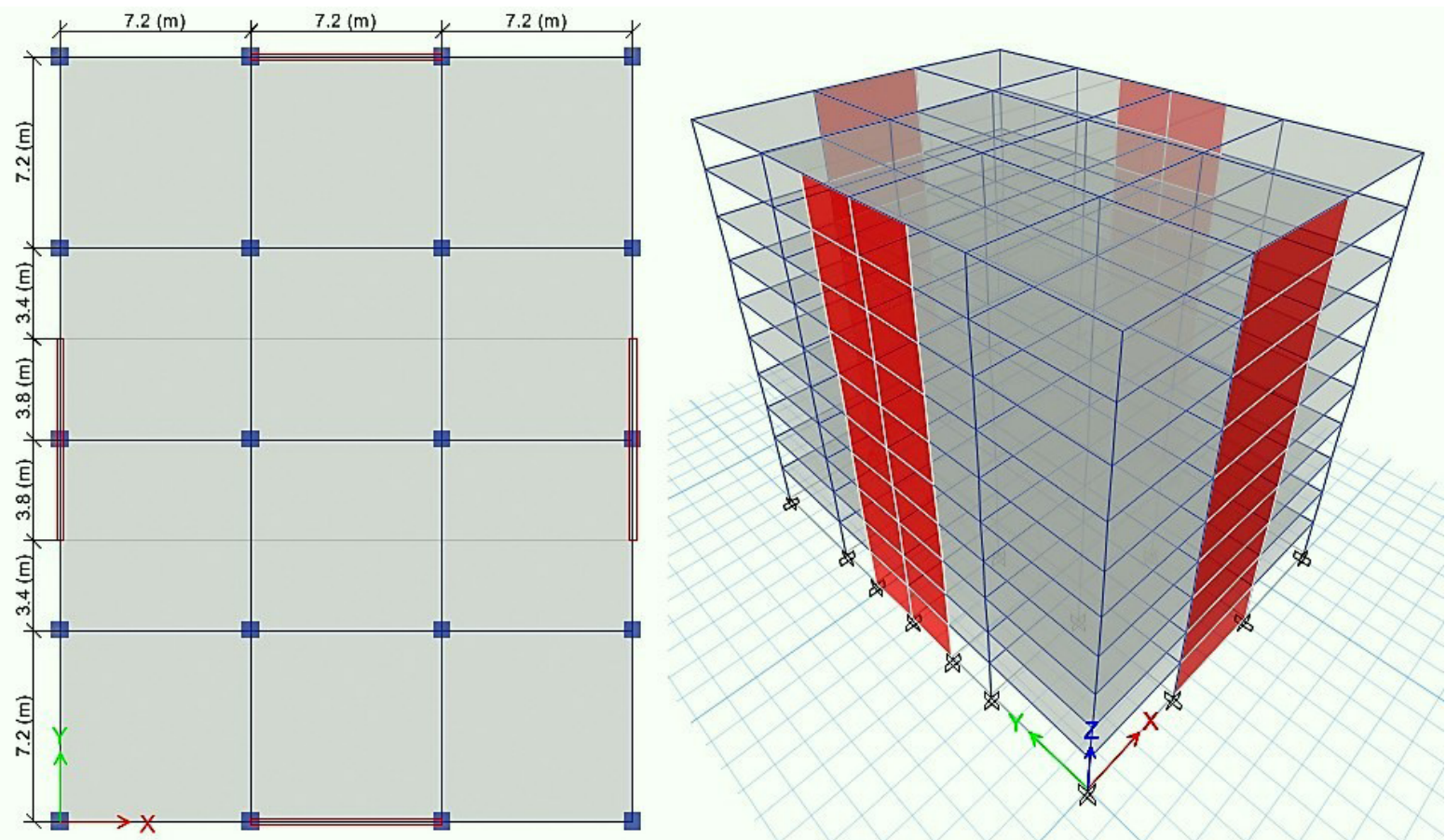

Figura 1. Planta y vista tridimensional de edificio. Fuente: Elaboración propia.

TABLA 1.

Características de los suelos D y E. Fuente: Elaboración propia.

\begin{tabular}{|c|c|c|c|c|c|c|}
\hline Suelo & $\begin{array}{c}\text { Módulo de } \\
\text { elasticidad } \\
\mathrm{E}(\mathrm{kPa})\end{array}$ & $\begin{array}{c}\text { Cohesión } \\
\mathrm{C}(\mathrm{kPa})\end{array}$ & $\begin{array}{c}\text { Ángulo de } \\
\text { fricción } \\
\Phi\left(^{\circ}\right)\end{array}$ & $\begin{array}{c}\text { Coeficiente } \\
\text { de Poisson } \\
\vartheta\end{array}$ & $\begin{array}{c}\text { Peso } \\
\text { especifico } \\
\gamma\left(\mathrm{kN} / \mathrm{m}^{3}\right)\end{array}$ & $\begin{array}{c}\text { Módulo de } \\
\text { cortante } \\
\mathrm{G}(\mathrm{kPa})\end{array}$ \\
\hline $\mathrm{D}$ & 23000 & 49.6 & 14.5 & 0.30 & 18.5 & 8846 \\
\hline $\mathrm{E}$ & 10000 & 50 & 10 & 0.35 & 16 & 1852 \\
\hline
\end{tabular}

TABLA 2.

Clasificación de los perfiles de suelo según NC 46: 2017 [1].

\begin{tabular}{|c|c|c|}
\hline Tipo de perfil de suelo & Descripción & Definición \\
\hline D & $\begin{array}{c}\text { Perfiles de suelos rígidos } \\
\text { de cualquier espesor que } \\
\text { cumpla con el criterio de } \\
\text { velocidad de la onda de cortante. }\end{array}$ & $180 \mathrm{~m} / \mathrm{s} \leq \mathrm{Vs} \leq 360 \mathrm{~m} / \mathrm{s}$ \\
\hline E & $\begin{array}{c}\text { Perfil de cualquier espesor } \\
\text { que cumpla el criterio de } \\
\text { velocidad de la onda de } \\
\text { cortante. Arcilla blanda. }\end{array}$ & Vs $<180 \mathrm{~m} / \mathrm{s}$ \\
\hline
\end{tabular}

El dimensionamiento de la losa de cimentación se obtuvo a partir de chequear la capacidad portante de la misma y los asentamientos absolutos y diferenciales que se producen. Las características de dicho elemento estructural se muestran en la tabla 3.

TABLA 3.

Dimensionamiento de la losa de cimentación para cada suelo analizado. Fuente: Elaboración propia.

\begin{tabular}{|c|c|c|c|c|}
\hline \multirow{2}{*}{ Suelo } & \multicolumn{4}{|c|}{ Losa de cimentación } \\
\cline { 2 - 5 } & Espesor (m) & $\begin{array}{c}\text { Lado menor } \\
(\mathrm{m})\end{array}$ & $\begin{array}{c}\text { Lado mayor } \\
(\mathrm{m})\end{array}$ & $\begin{array}{c}\text { Profundidad de } \\
\text { cimentación (m) }\end{array}$ \\
\hline $\begin{array}{c}\text { D } \\
\text { Suelo } \\
\text { rígido }\end{array}$ & 1 & 23 & 30 & 4 \\
\hline $\begin{array}{c}\text { E } \\
\text { Arcilla } \\
\text { blanda }\end{array}$ & 1 & 23 & 30 & 4 \\
\hline
\end{tabular}

\section{Materiales}

Todos los elementos estructurales son de hormigón armado con una densidad de $24 \mathrm{kN} / \mathrm{m}^{3}$. La resistencia a compresión ( $f^{\prime} \mathrm{c}$ ) es de $35 \mathrm{MPa}$ y su módulo de elasticidad de $2.8 \times 10^{4} \mathrm{MPa}$. El coeficiente de Poisson es 0.17.

\section{Condiciones de borde}

Las uniones columna-columna y viga-columna se consideran continuas. Las columnas y los tímpanos son continuos en toda la altura y las losas funcionan como un disco rígido.

En una primera variante, las bases de las columnas y tímpanos se consideraron empotradas. Al incluirse la IDSE, se emplearon resortes que representan el suelo en la modelación y a los que le fueron asignados un valor de rigidez en cuyo cálculo intervienen parámetros del suelo y de la estructura de cimentación.

\section{Cargas}

Cargas permanentes y de uso

Para la determinación de las cargas permanentes y de uso se emplearon las normas NC 283: 2003 [18] y NC 284: 2003 [19] respectivamente. En la tabla 4 se muestran los valores de sobrecarga anteriormente referenciados.

TABLA 4.

Sobrecargas permanentes y de uso. Fuente: Elaboración propia

\begin{tabular}{|c|c|c|}
\hline Elemento & Sobrecarga permanente $\left(\mathrm{kN} / \mathrm{m}^{2}\right)$ & $\begin{array}{c}\text { Sobrecarga de } \\
\text { uso }\left(\mathrm{kN} / \mathrm{m}^{2}\right)\end{array}$ \\
\hline Losa de entrepiso & 2.90 & 1.50 \\
\hline Losa de cubierta & 1.87 & 2.00 \\
\hline
\end{tabular}

\section{Carga sísmica}

El edificio se encuentra ubicado en el municipio Santiago de Cuba, correspondiente con la zona sísmica 5 según la clasifi- 
cación de la NC 46: 2017 [1]. La edificación no posee ninguna irregularidad en planta o elevación y no excede los $50 \mathrm{~m}$ de altura sobre la base por lo que la carga sísmica será determinada mediante la aplicación del Método Estático Equivalente, según los requisitos establecidos en la NC 46: 2017 [1].

La tabla 5 muestra los parámetros propios del sitio donde se encuentra ubicada la obra, fundamentales para la confección del espectro sísmico.

TABLA 5.

Sobrecargas permanentes y de uso. Fuente: Elaboración propia

\begin{tabular}{|c|c|c|c|c|c|c|}
\hline Provincia & Municipio & So(g) & Ss(g) & Sl(g) & TL(s) & Zona \\
\hline $\begin{array}{c}\text { Santiago } \\
\text { de Cuba }\end{array}$ & $\begin{array}{c}\text { Santiago } \\
\text { de Cuba }\end{array}$ & 0.513 & 1.035 & 0.428 & 6 & 5 \\
\hline
\end{tabular}

Donde $S_{S}$ es la ordenada espectral de periodo corto; $S_{1}$ la ordenada espectral con periodo de 1 s y $T_{L}$, el periodo de transición del periodo largo.

Se consideró el sismo de diseño como básico u ordinario, con un $10 \%$ de probabilidad de ser excedido en un periodo de 50 años de vida útil correspondiente a un periodo de retorno de 475 años. La edificación se ha clasificado como obra ordinaria con un nivel de protección sísmica D. Por tanto, según lo establecido en [1], el nivel de desempe- ño aceptado sería el operativo. Para determinar el nivel de diseño se debe emplear un factor de escalado $\mathrm{Kd}$, que en este caso resulta igual a 0.66 .

Las ordenadas espectrales $\left(\mathrm{S}_{\mathrm{a}}\right)$ se determinarán según el valor del periodo de vibración de la estructura $(\mathrm{T})$ según las expresiones de la (1) a la (4) contenidas en la NC 46: 2017 [1].

$$
\begin{aligned}
& S_{a}=S_{D S}\left(0,4+0,6 T / T_{0}\right) \text { para } \mathrm{T} \leq T_{0} \\
& S_{a}=S_{D S} \text { para } T_{0}<\mathrm{T} \leq T_{S} \\
& S_{a}=S_{D S} / T \text { para } T_{S}<\mathrm{T} \leq T_{L} \\
& S_{a}=\frac{S_{D 1^{*}} T_{L}}{T^{2}} \mathrm{~T}>T_{L}
\end{aligned}
$$

Siendo:

$S_{D S}$ la aceleración espectral de diseño para periodos cortos; $S_{D 1}$, la aceleración espectral de diseño para periodos largos; $T_{0}$ y $T_{S}$, los periodos de esquina del espectro junto con $T_{L}$.

En las figuras 2 y 3 se muestran los espectros de diseño para los suelos D y E respectivamente.

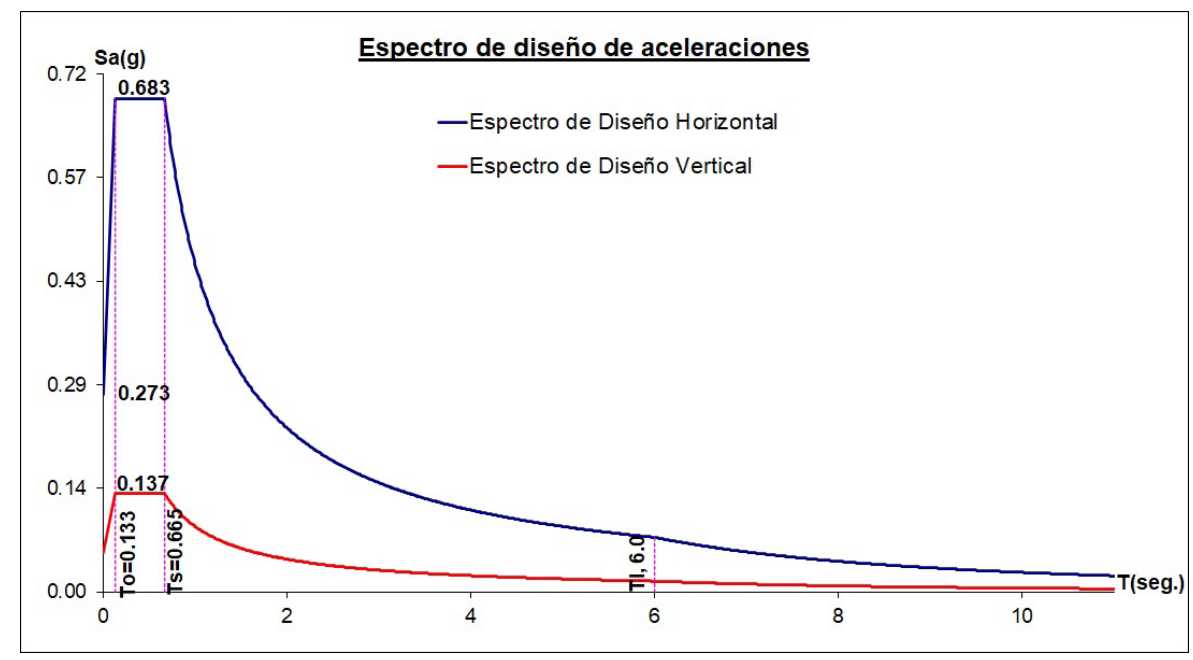

Figura 2. Espectro de diseño de aceleraciones horizontal y vertical para el suelo rígido (D). Fuente: Elaboración propia

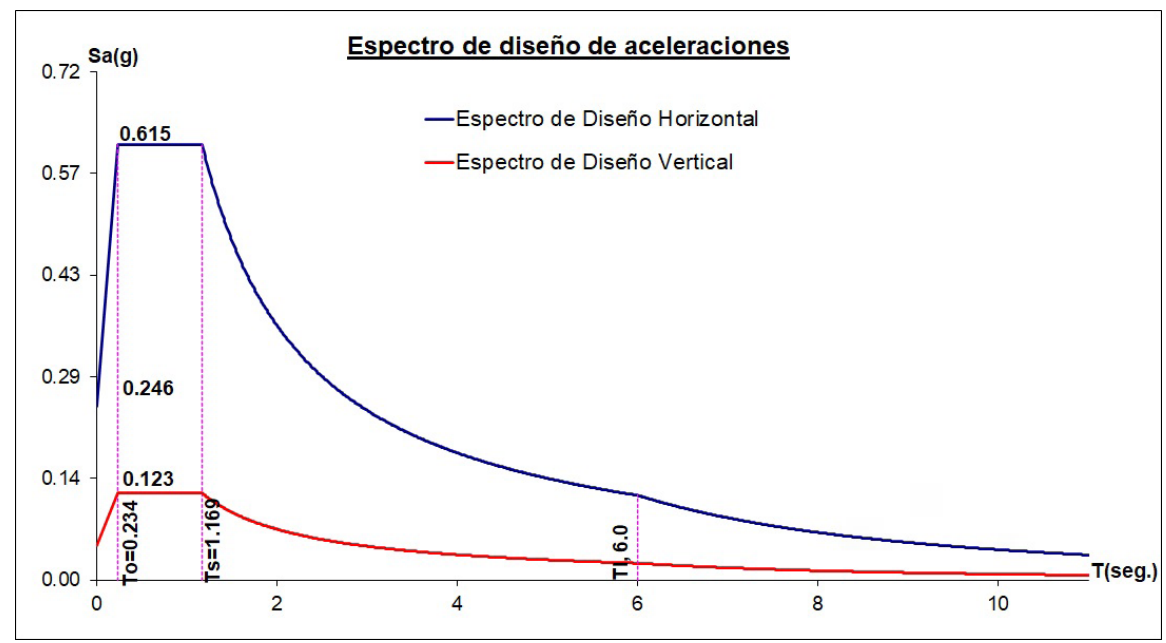

Figura 3. Espectro de diseño de aceleraciones horizontal y vertical para la arcilla blanda (E). Fuente: Elaboración propia. 


\section{Periodo propio de la estructura}

En un edificio sometido a cargas sísmicas, se considera como peso efectivo aquel que se encuentra físicamente ligado a la estructura. De acuerdo con [1] y [20] se incluirá, para la determinación del periodo de oscilación, la totalidad de las cargas permanentes y el 25\% de las cargas de utilización obteniéndose, como valores de este parámetro al considerar la base empotrada, 0.601 s y 0.618 s en las direcciones de análisis " $\mathrm{x}$ " y "y" respectivamente. Para ello se empleó el software de análisis y diseño ETABS V17.

\section{3.}

\section{REQUISITO COLUMNA FUERTE - VIGA DÉBIL}

El análisis estático no lineal Push - Over se lleva a cabo con el objetivo de evaluar el desempeño alcanzado por la estructura, evidenciado también en el orden y lugar de aparición de las rótulas plásticas, aspecto que se encuentra estrechamente vinculado al criterio de columna fuerte - viga débil. Por tanto, para implementar este método de análisis se debe, previamente, realizar el diseño estructural de los elementos columna y viga, el cual debe satisfacer la relación (5) planteada en [4]:

$$
\sum M_{n c} \geq(6 / 5) \sum M_{n b}
$$

Esta relación hace referencia a los momentos resistentes de las vigas $\left(M_{n b}\right)$ y columnas $\left(M_{n c}\right)$ que llegan a un mismo nudo. En la tabla 6 se muestra la relación obtenida para el caso de estudio según el tipo de suelo analizado, evidenciándose que, en todos los casos, dicha relación es mayor de 1,2, valor requerido en [4]. Para determinar estos valores se siguió el procedimiento establecido en [21].

TABLA 6.

Relación en la zona del nudo para los suelos analizados.

Fuente: Elaboración propia.

\begin{tabular}{|c|c|c|}
\hline Suelo & $\begin{array}{c}\Sigma M_{n c} / \Sigma M_{n b} \\
\text { (Para M2 en columna) }\end{array}$ & $\begin{array}{c}\Sigma M_{n c} / \Sigma M_{n b} \\
\text { (Para M3 en columna) }\end{array}$ \\
\hline Suelo rígido & 1.36 & 1.70 \\
\hline Arcilla blanda & 1.46 & 1.46 \\
\hline
\end{tabular}

4.

\section{PROCESO DE CÁLCULO PARA LA INCLUSIÓN EN EL MODELO DE LA IDSE}

Para la inclusión de la IDSE en el análisis se consideraron las funciones de impedancia de Pais \& Kausel incluidas en [16] y las formulaciones de Sargsian incluidas en [17]. Para ambos métodos se calcularon seis valores de rigidez equivalente correspondiente con cada uno de los grados de libertad. Se hace referencia a la parte dinámica de la interacción, ya que se analizan los esfuerzos y deformaciones que se inducen tanto en el suelo como en la estructura teniendo en cuenta no solo las cargas gravitacionales sino también la carga lateral de sismo [22]. Las formulaciones de la (6) a la (11), planteadas por [23] y recogidas en [24], corresponden con el cálculo de los términos de rigidez equivalente.

$$
\begin{aligned}
& K_{V}=\frac{G B}{1-V}\left(3,1 \lambda^{0,75}+1,6\right)\left(1+(0,25+0,25 / \lambda) \eta^{0,8}\right) \\
& \left.K_{X}=\frac{G B}{1-v}\left(6,8 \lambda^{0,65}+2,4\right)\left(1+\left(0,33+\frac{1,34}{1+\lambda}\right)\right) \eta^{0,8}\right) \\
& K_{y}=K_{X}+0,8 G B(\lambda-1)\left(1+\left(0,33+\frac{1,34}{1+\lambda}\right)+\eta^{0,8}\right) \\
& K_{r x}=\frac{G B^{3}}{1-v}(3,2 \lambda+0,8)\left(1+\eta+\frac{1,6}{0,35+\eta} \eta^{2}\right) \\
& K_{r y}=\frac{G B^{3}}{1-v}\left(3,73 \lambda^{2,4}+0,27\right)\left(1+\eta+\frac{1,6}{0,35+\lambda^{4}} \eta^{2}\right) \\
& K_{t}=16 G R^{3}\left(4,25 \lambda^{2,45}+0,46\right)\left(1+\left(1,3+\frac{1,3}{\lambda}\right) \eta^{0,9}\right)
\end{aligned}
$$

Donde: $\mathrm{K}_{\mathrm{v}}$, rigidez equivalente en la dirección vertical; $\mathrm{K}_{\mathrm{x}}$, rigidez equivalente para cada una de las direcciones horizontales de análisis; $\mathrm{K}_{\mathrm{r}}$, dos rigideces equivalentes en rotación con respecto a los ejes centroidales de la base perpendicular a cada dirección que se analiza; $\mathrm{K}_{\mathrm{t}}$, rigidez equivalente en torsión con respecto al eje vertical centroidal de la base; $G$, módulo de cortante; $v$, coeficiente de Poisson; $\mathrm{B}$, lado menor de la cimentación; $\mathrm{R}$, Radio equivalente; $\lambda=\mathrm{L} / \mathrm{B} ; \eta=\mathrm{E} / \mathrm{B}$.

Las formulaciones para la inclusión de la IDSE mediante modelo de Sargsian incorporan parámetros cuasi - estáticos de rigidez de la base de cimentación que se ajustan para plateas de cimentación, obteniéndose las expresiones de la (12) a la (16):

$$
\begin{aligned}
& K_{x}=K_{y}=\frac{28,8 \rho \mathrm{C}_{2}^{2}}{\sqrt{\pi}(7-8 \mu)} \sqrt{A} \\
& K_{Z}=\frac{4 \rho \mathrm{C}_{2}^{2}}{\sqrt{\pi}(1-\mu)} \sqrt{A} \\
& K_{\varphi x}=\frac{8,52 \rho \mathrm{C}_{2}^{2}}{\sqrt{\pi}(1-\mu)} \frac{I_{x}}{\sqrt{A}} \\
& K_{\varphi y}=\frac{8,52 \rho \mathrm{C}_{2}^{2}}{\sqrt{\pi}(1-\mu)} \frac{I_{y}}{\sqrt{A}} \\
& K_{\psi z}=\frac{8,52 \rho \mathrm{C}_{2}^{2}}{\sqrt{\pi}(1-\mu)} \frac{I_{z}}{\sqrt{A}}
\end{aligned}
$$

Siendo: $A$, área de la platea de cimentación; $I_{x}, I_{y}$, momentos de inercia de la losa de cimentación respecto a los ejes $\mathrm{X}$ y Y; $I_{y}$, momento de inercia de la losa de cimentación respecto al eje vertical $Z$ (momento polar de inercia) y $C_{2}$ la velocidad de propagación de la onda transversal. De acuerdo con la concepción de semiespacio elástico, este último parámetro se puede calcular mediante la expresión (17):

$C_{2}=\sqrt{\frac{E}{2(1+\mu) \rho}}$ 
TABLA 7.

Valores de rigidez equivalente para los suelos analizados. Formulaciones de Pais \& Kausel. Fuente: Elaboración propia.

\begin{tabular}{|c|c|c|c|c|c|c|}
\hline \multirow{2}{*}{ Suelo } & \multicolumn{5}{|c|}{ Rigideces equivalentes } \\
\cline { 2 - 7 } & $\mathrm{Kx}(\mathrm{kN} / \mathrm{m})$ & $\mathrm{Ky}(\mathrm{kN} / \mathrm{m})$ & $\mathrm{Kv}(\mathrm{kN} / \mathrm{m})$ & $\operatorname{Krx}(\mathrm{kNm})$ & $\mathrm{Kry}(\mathrm{kNm})$ & $\mathrm{Kt}(\mathrm{kNm})$ \\
\hline Suelo rígido & 3272718 & 3340949 & 1621053 & 799432506 & 1176708995 & 4794331128 \\
\hline Arcilla blanda & 1475621 & 504188 & 730909 & 360452659 & 530561221 & 2007288395 \\
\hline
\end{tabular}

TABLA 8 .

Valores de rigidez equivalente para los suelos analizados. Formulaciones de Sargsian. Fuente: Elaboración propia.

\begin{tabular}{|c|c|c|c|c|c|c|}
\hline \multirow{2}{*}{ Suelo } & \multicolumn{5}{|c|}{ Rigideces equivalentes } \\
\cline { 2 - 7 } & $\mathrm{Kx}(\mathrm{kN} / \mathrm{m})$ & $\mathrm{Ky}(\mathrm{kN} / \mathrm{m})$ & $\mathrm{Kv}(\mathrm{kN} / \mathrm{m})$ & $\mathrm{Krx}(\mathrm{kNm})$ & $\mathrm{Kry}(\mathrm{kNm})$ & $\mathrm{Kt}(\mathrm{kNm})$ \\
\hline Suelo rígido & 820801 & 820801 & 749144 & 119675818 & 70342786 & 89210613 \\
\hline Arcilla blanda & 376381 & 376381 & 337778 & 53960111 & 31716554 & 40223786 \\
\hline
\end{tabular}

En las tablas 7 y 8 se muestran los valores de rigidez calculados para los suelos analizados de acuerdo con las formulaciones de Pais \& Kausel y de Sargsian respectivamente.

A partir de lo anterior, se realiza el análisis estático no lineal push - over para las dos condiciones de apoyo: base empotrada y considerando la IDSE con el empleo de los métodos basados en las formulaciones de Pais \& Kausel y Sargsian para el cálculo de las rigideces equivalentes en su implementación, para los dos tipos de suelos presentados.

\section{Resultados y discusión}

Los efectos de la IDSE se evidencian en las modificaciones que sufren los parámetros que rigen la respuesta estructural de la edificación, específicamente el periodo de oscilación, la distribución de fuerzas sísmicas por niveles, los desplazamientos y derivas, el punto y nivel de desempeño alcanzado y el orden y lugar de aparición de las rotulas plásticas. Para cada caso se analizan los resultados obtenidos.

\section{Periodo de oscilación (T)}

La tabla 9 muestra los valores de periodo fundamental de oscilación de la estructura según condición de apoyo analizada, formulaciones empleadas para el cálculo de la IDSE y tipo de suelo. Dichos valores corresponden a la combinación $G+0.25$ $\mathrm{Q}$, siendo G la carga permanente y Q la carga de utilización.

TABLA 9.

Periodo fundamental de oscilación de la estructura. Fuente: Elaboración propia.

\begin{tabular}{|c|c|c|}
\hline Condición de apoyo & Suelo & $\begin{array}{l}\text { Periodo fundamental } \\
\text { de la estructura (s) }\end{array}$ \\
\hline \multirow{2}{*}{ Suelo rígido } & \multirow{2}{*}{ - } & 0.618 \\
\hline & & 0.618 \\
\hline \multirow{2}{*}{$\begin{array}{c}\text { IDSE } \\
\text { (Pais\& Kausel) }\end{array}$} & Suelo Rígido & 0.632 \\
\hline & Arcilla blanda & 0.648 \\
\hline \multirow{2}{*}{$\begin{array}{c}\text { IDSE } \\
\text { (Sargsian) }\end{array}$} & Suelo Rígido & 0.669 \\
\hline & Arcilla blanda & 0.701 \\
\hline
\end{tabular}

Los periodos obtenidos, independientemente del método empleado, se encuentran en los rangos recomendados en [25], [26] atendiendo a la tipología estructural de la edificación y no implican el peligro de existencia de correlaciones modales que traen consigo la amplificación de las ondas sísmicas que pueden llevar al colapso o provocar daños estructurales severos.

El periodo de oscilación se modifica al incluir la IDSE en el análisis dado el incremento que tiene lugar en la flexibilidad del suelo al no considerar la base perfectamente empotrada. Un edificio que yace sobre suelo rígido se comportará con tendencia a un voladizo y tendrá un período de oscilación menor que uno que tenga su cimentación en un suelo blando, puesto que el conjunto subestructura-superestructura se tornará más flexible y el período se incrementa al comportarse como parcialmente empotrado debido a las deformaciones que se van a producir en dicho empotramiento.

Lo anterior se evidencia en los resultados obtenidos debido a que el periodo de oscilación se incrementa independientemente del método aplicado para la inclusión de la IDSE y para ambos tipos de suelos analizados. Se destaca que el mayor incremento, comparado con la base empotrada, alcanza el $16.64 \%$ al emplear las formulaciones de Sargsian para la arcilla blanda, debido al módulo de elasticidad, que al ser menor que el del suelo rígido, origina un incremento en la oscilación de la edificación.

\section{Desplazamiento en el tope y derivas}

En la figura 4 se muestran los valores de desplazamiento en el tope de la edificación para cada uno de los modelos analizados según la condición de apoyo de la base y las formulaciones empleadas para la inclusión de la IDSE, para ambos tipos de suelo. La combinación pésima está dada para $\mathrm{G}+\mathrm{Q}+\mathrm{Sv}+\mathrm{Shx}$ siendo G la carga permanente; $\mathrm{Q}$ la carga de utilización; Sv, la componente vertical de la carga de sismo y Shx, la componente horizontal de la carga de sismo en el sentido X de la edificación.

En todos los casos, el desplazamiento en el tope aumenta al considerar la IDSE. El mayor por ciento de incremento, con relación a la base empotrada, tiene lugar cuando se incluye este fenómeno a través de las formulaciones de Sargsian y para el 
G+Q-Sv+Shx

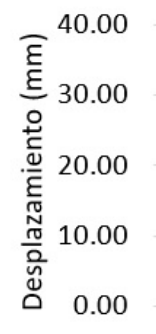

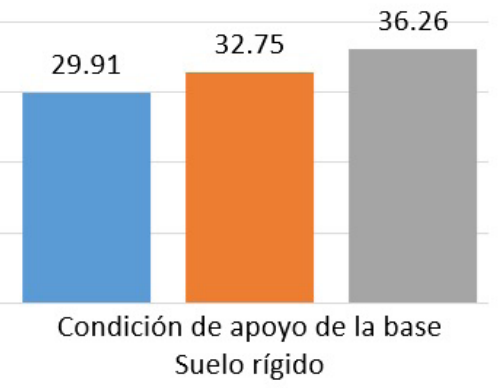

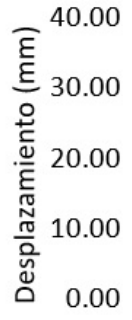

n Base Empotrada

\section{G+Q-Sv+Shx}

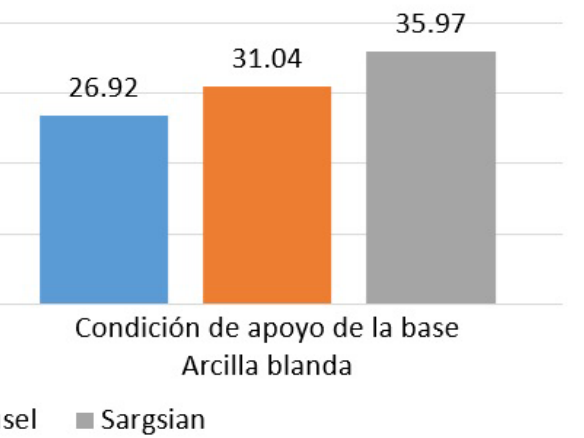

Figura 4. Desplazamiento en el tope de la edificación para todos los casos analizados. Fuente: Elaboración propia.

$\mathrm{G}+\mathrm{Q}-\mathrm{Sv}+\mathrm{Sh} \mathbf{x}$

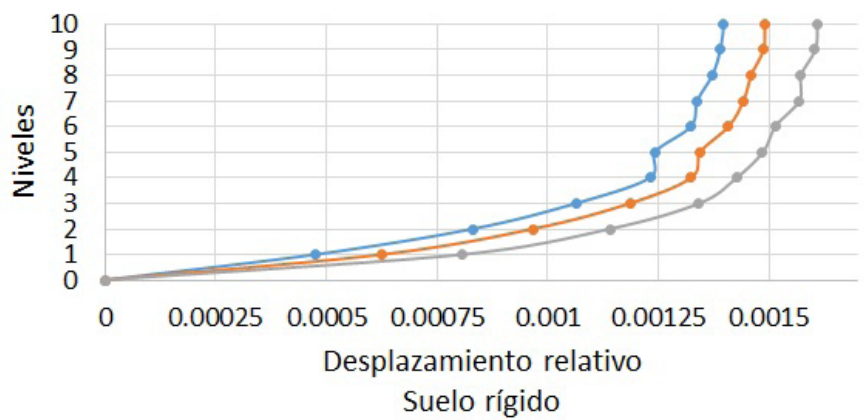

G+Q-Sv+Shx

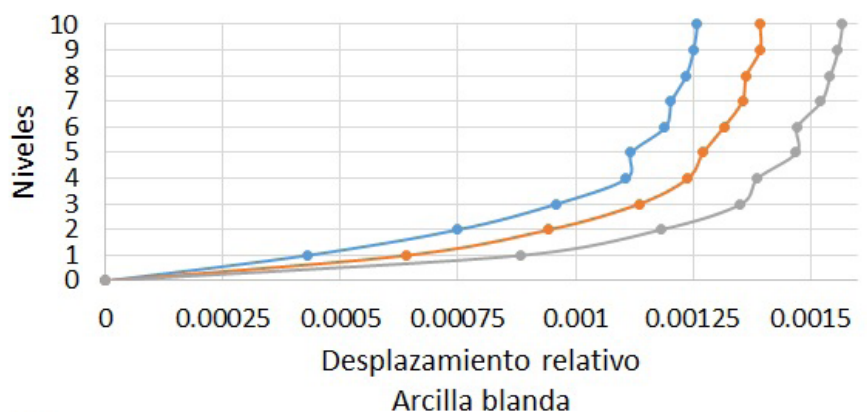

$\multimap$ Base Empotrada $\multimap$ Pais \& Kausel $\multimap$ Sargsian

Figura 5. Derivas por piso. Fuente: Elaboración propia.

Suelo rígido

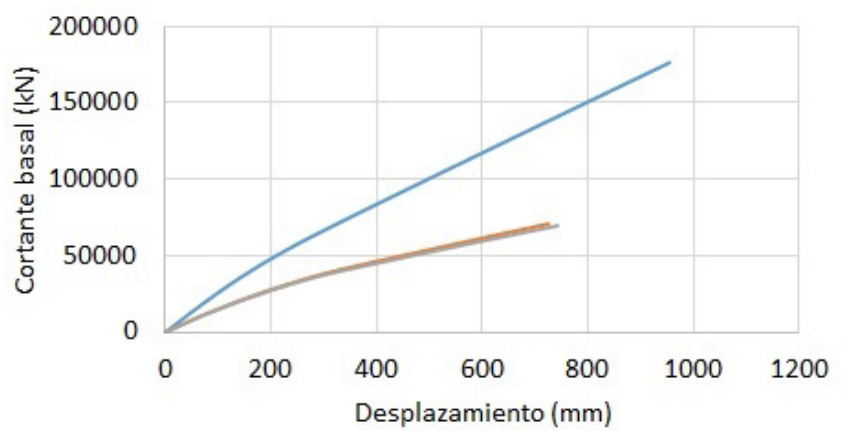

\section{Arcilla blanda}

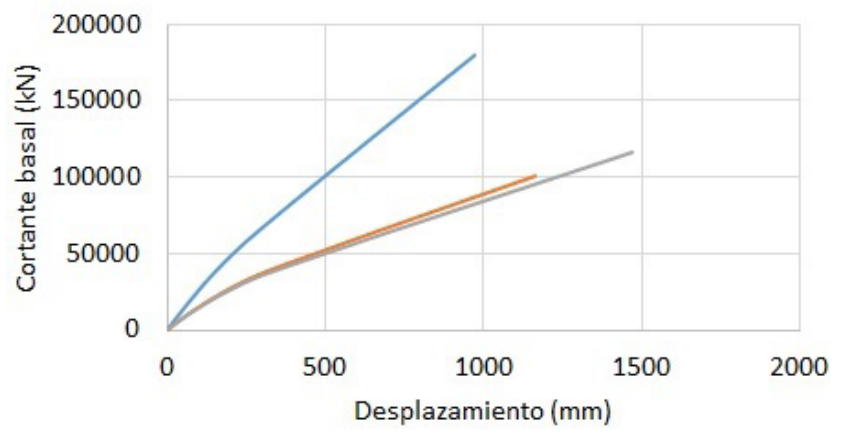

— Base Empotrada — IDSE (Pais \& Kausel) —IDSE (Sargsian)

Figura 6. Curvas de capacidad. Fuente: Elaboración propia.

suelo formado por arcillas blandas, siendo dicho incremento del $33.62 \%$.

En el caso de las derivas el comportamiento es similar. En la figura 5 se muestran las derivas por piso para cada uno de los modelos analizados según la condición de apoyo de la base y las formulaciones empleadas para la inclusión de la IDSE, para ambos tipos de suelo.

Se aprecia un aumento de los valores de deriva en todos los niveles cuando se considera la IDSE destacándose el mayor incremento cuando se emplean las formulaciones de Sargsian. El mayor por ciento de variación tiene lugar siempre en el primer nivel, con un incremento del $105.83 \%$, con relación a la base empotrada, obtenido para el suelo formado por arcillas blandas al incluir la IDSE mediante el método de Sargsian. Estos incrementos en las derivas que tienen lugar al considerar la IDSE y que son obviados cuando se trata la base como empotrada, implican la posible ocurrencia de fallos en los entrepisos por la aparición de articulaciones plásticas en las columnas antes que en la vigas y por tanto afectar la ductilidad general del conjunto, pues no se realiza un análisis realista de la relación de rigidez viga-columna requerida de los pórticos del sistema que garantice el comportamiento de columna fuerte-viga débil.

\section{Curvas de capacidad}

En la figura 6 se muestran las curvas de capacidad obtenidas para ambas condiciones de apoyo de la base, atendiendo a los 
dos tipos de suelo analizados y se representa la respuesta no lineal de la estructura y la resistencia de ésta ante la acción sísmica.

Se aprecia en la figura 6 cómo, al incluir la IDSE e independientemente del método empleado para ello, los desplazamientos aumentan en mayor medida para pequeños incrementos de cortante basal si se compara con la base empotrada. Lo anterior evidencia una reducción de la rigidez lateral de la edificación. Los casos en los que se consideró la base empotrada demuestran una mayor rigidez lateral al desarrollar menores incrementos de desplazamientos ante la carga sísmica a la que se encuentra sometido. Resultados similares fueron obtenidos por [27].

\section{Cortante y desplazamiento de fluencia}

El valor de cortante de fluencia de la estructura marca el inicio del rango plástico en el comportamiento de ésta. Este valor se encuentra asociado al cálculo de los factores de reducción por ductilidad y sobrerresistencia incluidos en las normativas referidas al diseño sismorresistente [28]. Por su parte el desplazamiento de fluencia, provocado por el cortante de fluencia, interviene en la determinación de la ductilidad global alcanzada por la estructura [29]. En la figura 7 se muestran los valores de estos dos parámetros, atendiendo al tipo de suelo y a la condición de apoyo de la base.

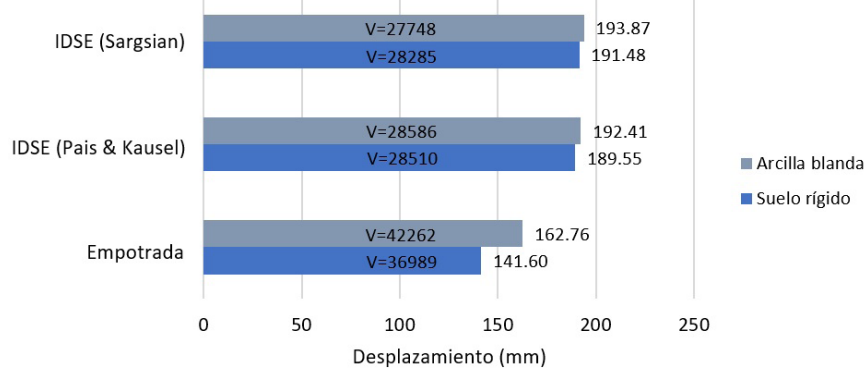

Figura 7. Cortante y desplazamiento de fluencia. Fuente: Elaboración propia.

En los resultados mostrados en la figura 7 se aprecia que el cortante de fluencia de la estructura disminuye al incluir la IDSE en los modelos. Esto se debe a que la fluencia efectiva de la estructura con base empotrada depende, principalmente, de la fluencia de las vigas que incursionan en el rango inelástico; mientras que en los casos en los que se ha introducido la IDSE la pérdida de rigidez está regida por la fluencia de las columnas de planta baja. En los casos en que el trabajo inelástico se concentre en las vigas, la capacidad de deformación está dada mayoritariamente por el trabajo conjunto de estas antes de alcanzar la fluencia global de la edificación. Lo anterior significa que, al incluir la IDSE, la capacidad de deformación de la estructura se reduce. Resultados similares se obtienen en [12].

En los casos de estudio, las mayores diferencias en cuanto al desplazamiento de fluencia con relación a la base empotrada se reportan al introducir la IDSE mediante las formulaciones de Sargsian y para el suelo rígido, siendo estas del $35.23 \%$. Con relación al cortante basal de fluencia, la mayor diferencia tiene lugar para el caso del suelo de arcilla blanda e igual método empleado para la introducción de la IDSE, siendo esta del $34.34 \%$.
Formación de rótulas plásticas y mecanismo de colapso

En las figuras 8, 9 y 10 se observa cómo al considerar la base empotrada las rótulas plásticas se han formado solamente en las vigas de la edificación, mientras que al incluir la IDSE, estas comienzan a aparecer en las bases de las columnas.
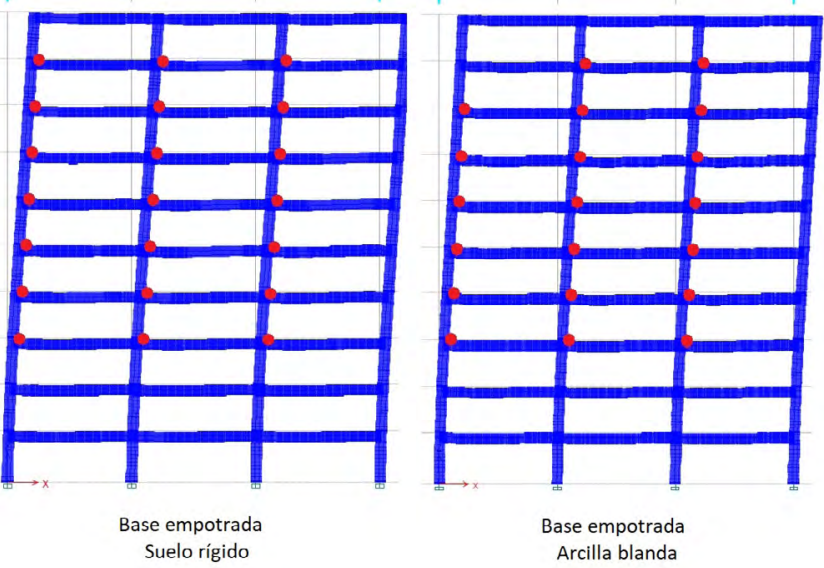

Figura 8. Formación de rótulas plásticas. Base empotrada. Fuente: ETABS V17.
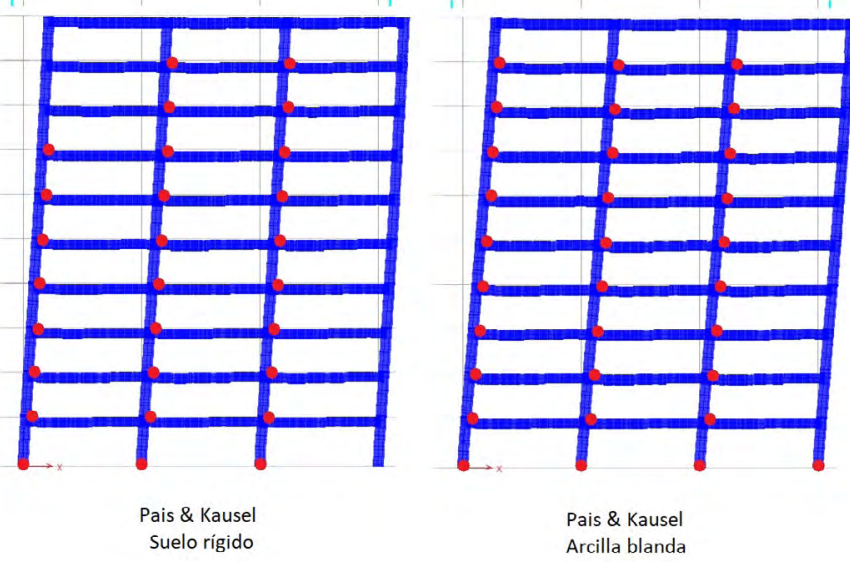

Figura 9. Formación de rótulas plásticas. Pais \& Kausel. Fuente: ETABS V17.
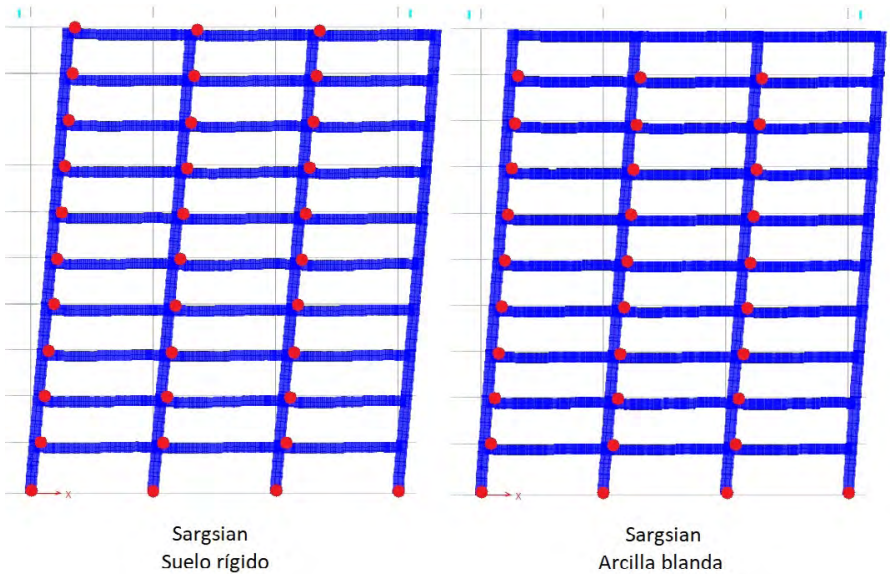

Figura 10. Formación de rótulas plásticas. Sargsian. Fuente: ETABS V17.

El mecanismo de colapso generado estará en dependencia del orden y aparición de las rótulas plásticas. Es recomendable que estas ocurran primeramente en las vigas, ya que la degra- 
TABLA 10.

Valores de desplazamiento en el tope y cortante basal para los que aparece la primera rótula en columnas. Fuente: Elaboración propia.

\begin{tabular}{|c|c|c|c|}
\hline \multirow{2}{*}{ Suelo } & $\begin{array}{c}\text { Condición de } \\
\text { apoyo de la base }\end{array}$ & Elemento & $\begin{array}{c}\text { Vesplazamiento } \\
(\mathbf{m m})\end{array}$ \\
\hline \multirow{3}{*}{ Suelo rígido } & Empotrada & Columna & 87.21 \\
\cline { 2 - 4 } & IDSE (Pais \& Kausel) & Columna & 54.91 \\
\cline { 2 - 4 } & IDSE (Sargsian) & Columna & 56.09 \\
\hline \multirow{2}{*}{ Arcilla blanda } & Empotrada & Columna & 87.21 \\
\cline { 2 - 4 } & IDSE (Pais \& Kausel) & Columna & 55.71 \\
\cline { 2 - 4 } & IDSE (Sargsian) & Columna & 5714 \\
\hline
\end{tabular}

dación de la resistencia en las columnas sucede más rápido debido a la presencia de la carga axial.

En la tabla 10 se expone el valor de cortante basal y desplazamiento en el tope correspondiente, para los que se desarrolla la primera rótula plástica en las columnas para cada uno de los modelos analizados.

Los mayores porcientos de disminución del cortante basal asociado a la formación de la primera rótula plástica en columnas se obtienen en el suelo formado por arcilla blanda siendo estos del $61.58 \%$ y $63.09 \%$ para los modelos en que se empleó Pais \& Kausel y Sargsian respectivamente. Dichas diferencias son similares a las obtenidas en el suelo rígido.

La formación de rótulas plásticas en vigas permite una mayor disipación de energía sin incurrir en grandes daños. El hecho de que, como se muestra en la tabla 11, la aparición de la primera articulación plástica en columnas al incluir la IDSE tenga lugar para un valor de cortante basal aproximadamente un $60 \%$ menor que para la base empotrada, implica que la pérdida de rigidez en estos elementos comenzará antes de lo previsto según el diseño convencional que considera en el análisis la base empotrada, lo que puede traer consigo una modificación en el mismo.

La aparición continua de rótulas plásticas en las columnas de un mismo nivel trae consigo la formación del mecanismo de colapso conocido como "mecanismo de piso" o "piso blando", forma de fallo común en edificaciones ubicadas en zonas de alto riesgo sísmico. Se analiza el valor de cortante basal para el cual se genera dicho mecanismo, determinado por la formación de rótulas en ambos extremos de todas las columnas de un mismo nivel.

En las figuras 11, 12 y 13 se muestra la formación del mecanismo de colapso conocido como piso blando. En ellas se evidencia que, en los casos en los que la base se encuentra empotrada, existe un predominio de la deformación por flexión general, mientras que en los restantes casos predomina la deformación por cortante general.

En la tabla 11 se exponen los valores de cortante basal que generan el mecanismo de colapso para cada caso, así como el desplazamiento que se produce.

La aparición de este fallo local tiene lugar para valores inferiores de cortante basal al incluir la IDSE, generando también mayores desplazamientos con respecto al modelo con base empotrada. Esto implica que al no incorporar la IDSE en el análisis de una edificación ante carga sísmica se incurriría en daños no considerados en el diseño estructural, generándose el colapso antes de lo previsto. Los mayores por cientos de
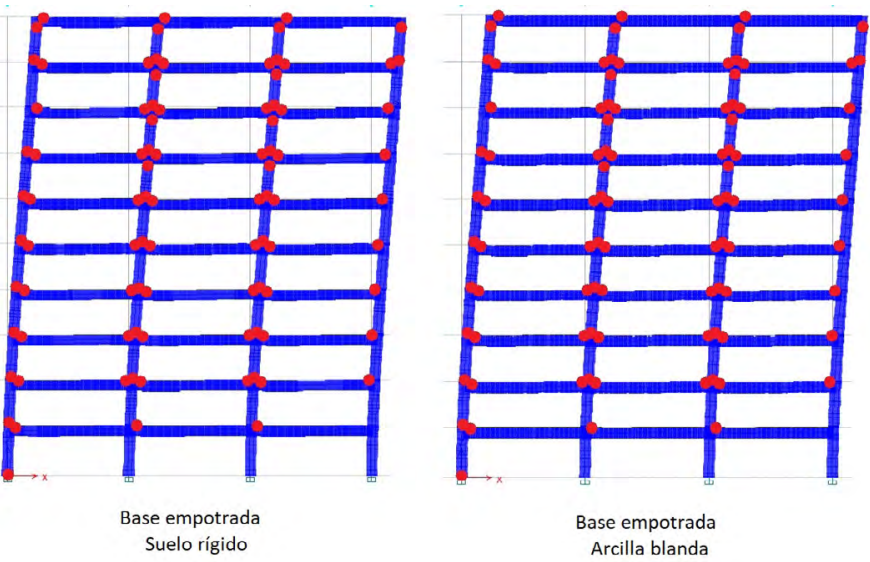

Figura 11. Mecanismo de piso blando. Base empotrada. Fuente: ETABS V17.

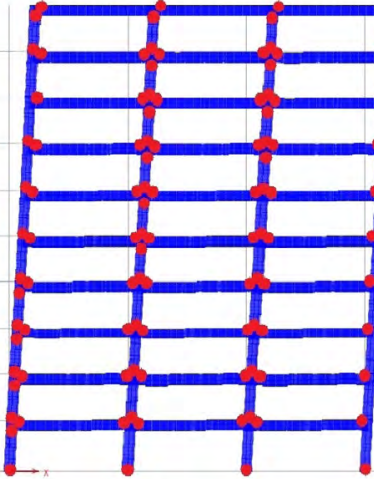

Pais \& Kause Suelo rígido

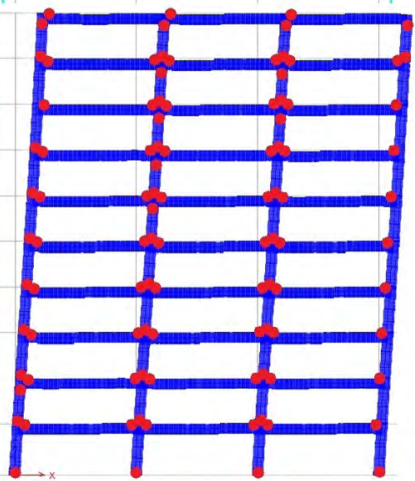

Pais \& Kausel Arcilla blanda
Figura 12. Mecanismo de piso blando. Pais \& Kausel. Fuente: ETABS V17.
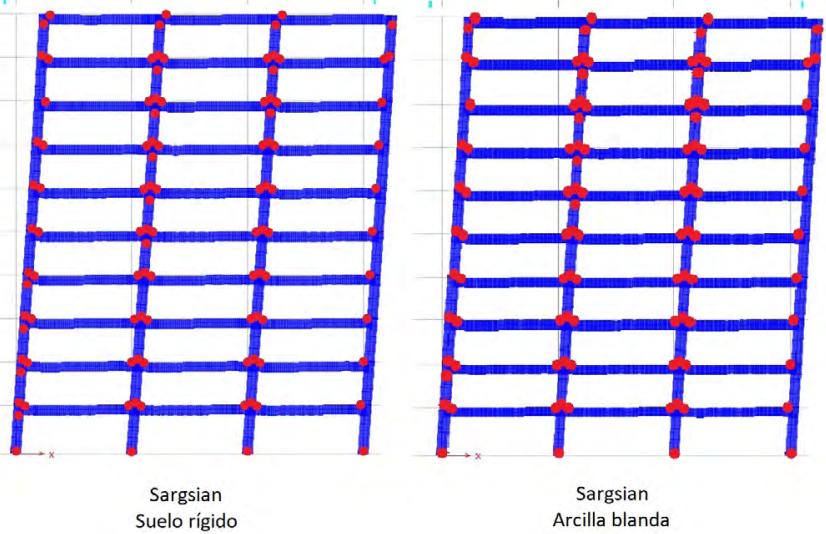

Figura 13. Mecanismo de piso blando. Sargsian. Fuente: ETABS V17. 
TABLA 11.

Cortante basal correspondiente con la generación de un “mecanismo de piso”. Fuente: Elaboración propia.

\begin{tabular}{|c|c|c|c|}
\hline \multirow{2}{*}{ Suelo } & Condición de apoyo de la base & Cortante basal (kN) & Desplazamiento (mm) \\
\hline \multirow{3}{*}{ Suelo rígido } & Empotrada & 47949 & 199.39 \\
\cline { 2 - 4 } & IDSE (Pais \& Kausel) & 35842 & 273.44 \\
\cline { 2 - 4 } & IDSE (Sargsian) & 34117 & 262.28 \\
\hline \multirow{3}{*}{ Arcilla blanda } & Empotrada & 47949 & 199.39 \\
\cline { 2 - 4 } & IDSE (Pais \& Kausel) & 32056 & 245.86 \\
\cline { 2 - 4 } & IDSE (Sargsian) & 30986 & 247.64 \\
\hline
\end{tabular}

diferencia en el cortante basal al incorporar la IDSE tienen lugar para el suelo de arcilla blanda, dada la mayor deformabilidad que este presenta respecto al suelo rígido, siendo estos del $36.05 \%$ y del $42.42 \%$ para las formulaciones de Pais \& Kausel y Sargsian respectivamente.

\section{Punto de desempeño}

La inclusión de la IDSE trae como consecuencia que el punto de desempeño se modifique, incurriendo en niveles asociados a daños estructurales de mayor gravedad. Esta tendencia ha sido evidenciada en [30]. En las figuras 14, 15 y 16 se muestra el punto de desempeño obtenido según [31] para ambos suelos según la condición de apoyo de la base.

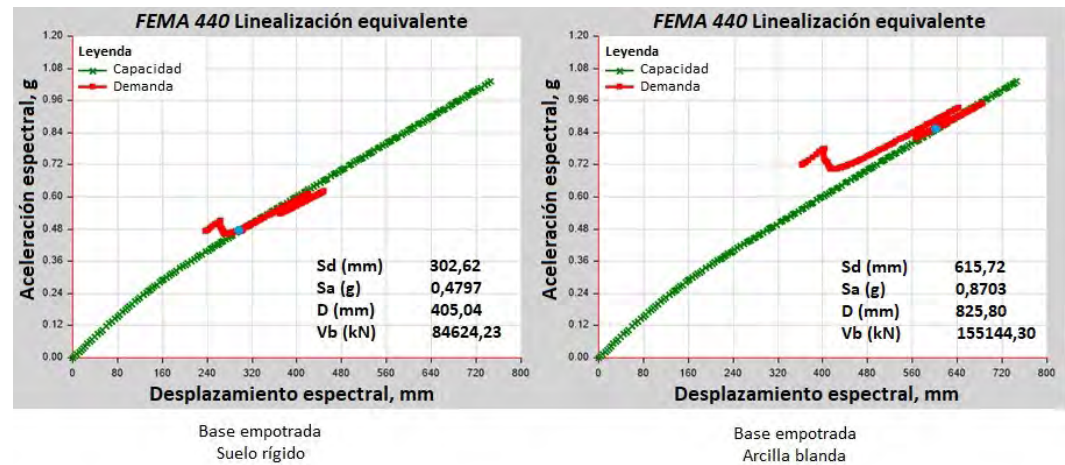

Figura 14. Curvas de capacidad y demanda en formato ADRS. Punto de desempeño. Base empotrada. Fuente: ETABS V17.

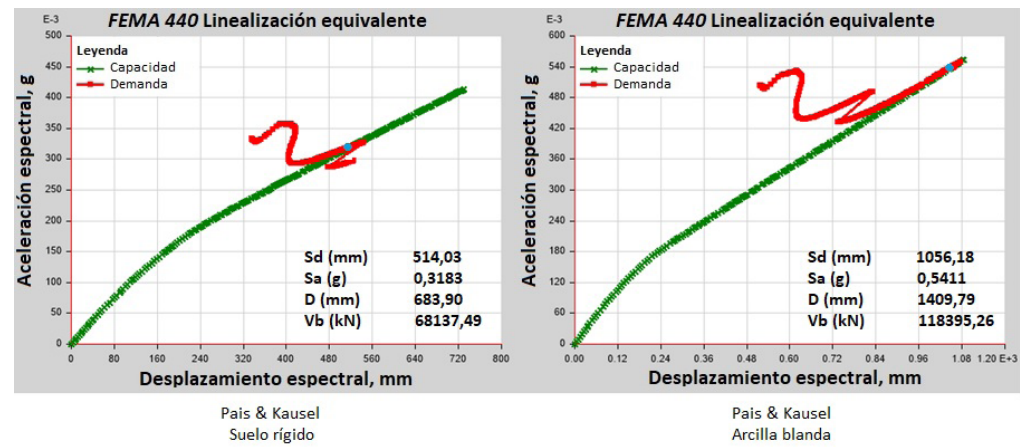

Figura 15. Curvas de capacidad y demanda en formato ADRS. Punto de desempeño. Pais \& Kausel. Fuente: ETABS V17.

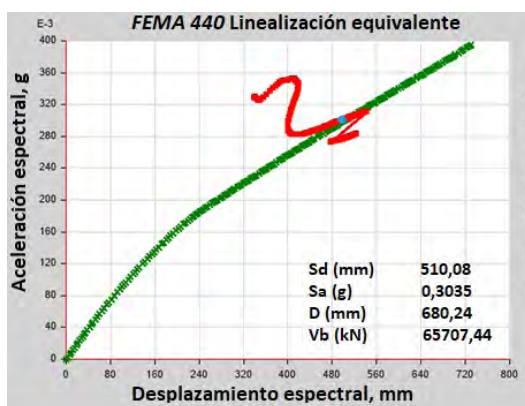

Sargsian

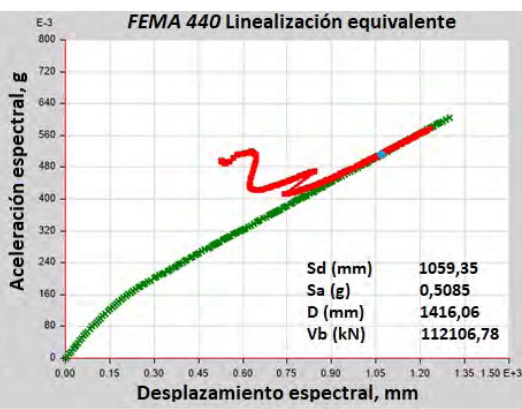

Sargsian

Figura 16. Curvas de capacidad y demanda en formato ADRS. Punto de desempeño. Sargsian. Fuente: ETABS V17. 


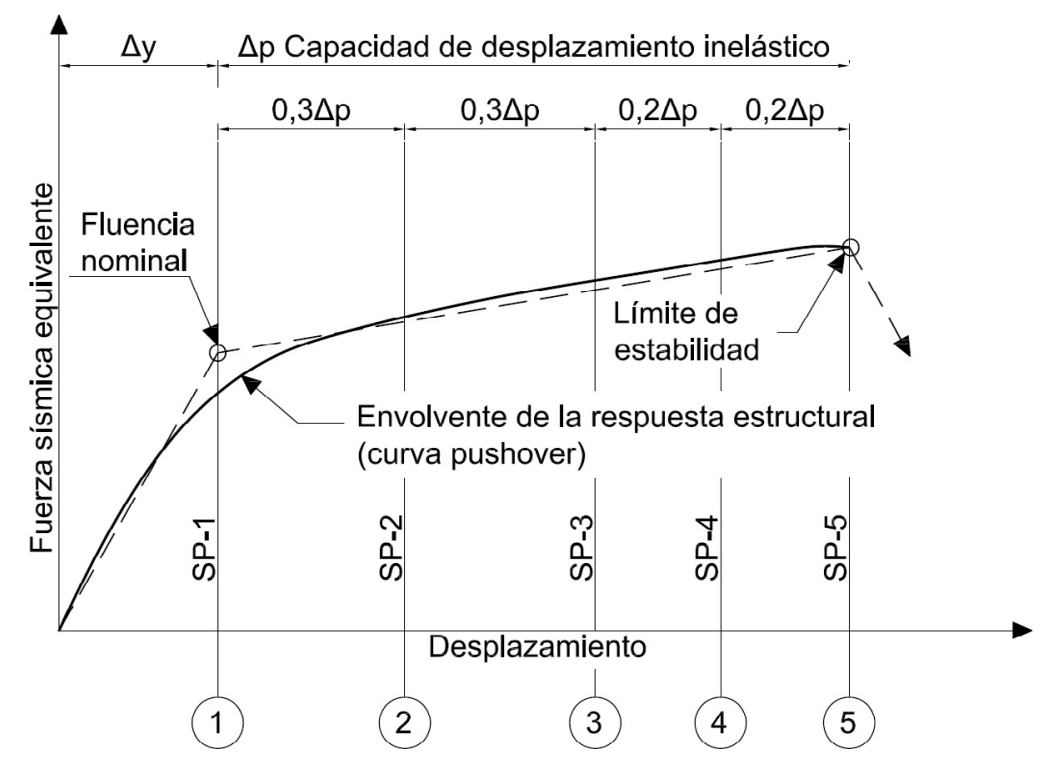

Figura 17. Sectorización de la curva de capacidad. Fuente: [10].

El punto de desempeño se desplaza hacia la derecha al incluir la IDSE, independientemente del método empleado para ello. En la medida en la que esto ocurre, el desplazamiento alcanzado por la edificación comienza a incluirse en rangos que definen niveles de desempeño asociados a mayores daños en la edificación. Los mayores incrementos en el desplazamiento máximo obtenido tienen lugar para la arcilla blanda, siendo estos del $70.72 \%$ y del $71.48 \%$ al incorporar la IDSE mediante los modelos de País \& Kausel y de Sargsian respectivamente. De igual manera se destacan las modificaciones que tienen lugar para el suelo rígido, siendo estas del $68.85 \%$ y del $67.94 \%$ para dichos modelos.

\section{Nivel de desempeño}

Para la obtención del nivel de desempeño se procede a la sectorización de la curva de capacidad de acuerdo con lo planteado en [10]. En este documento se establecen los niveles de desempeño a partir de rangos de desplazamiento lineal y no lineal y de acuerdo con el nivel de daño esperado en la edificación. En la NC 46: 2017 [1] no se plantea una sectorización de dicha curva pero sí se definen niveles de desempeño, con lo cual es posible establecer una equivalencia entre los documentos ya mencionados. En correspondencia con esto, los niveles totalmente operacional, operacional, seguridad de vida y pre-colapso de SEAOC [10] son equivalentes con los niveles ocupación inmediata, operativo, seguridad de vida y prevención de colapso de la NC 46: 2017 [1] respectivamente. A diferencia de [10], [1] no contempla el nivel de desempeño colapso. Ambos documentos recomiendan que las edificaciones tengan un desempeño determinado según el tipo de obra e importancia de esta y la intensidad del sismo que incide sobre ella. La figura 17 muestra el esquema de sectorización de la curva de capacidad propuesto en [10].

Como se aprecia en la figura 17, la sectorización de la curva está en función del desplazamiento elástico y la capacidad de desplazamiento inelástico.

En las figuras 18, 19 y 20 se observa la sectorización de las curvas de capacidad correspondiente a los casos analizados en las que se indica el nivel de desempeño alcanzado en cada uno de ellos.

Para ambos suelos, e independientemente del método empleado para la consideración de la IDSE, el nivel de desempeño se modifica haciendo que la estructura incursione en los rangos de pre-colapso para el caso del suelo rígido y colapso para la arcilla blanda respectivamente, por lo que la edificación incurre en daños de mayor envergadura. Se destaca que, al igual que el resto de los parámetros evaluados, las mayores modificaciones tienen lugar en el suelo formado por arcilla blanda. Las modificaciones obtenidas al introducir la IDSE en la localización del punto de desempeño difieren poco entre un método y otro, obteniéndose por cientos de variación inferiores al 1\% en cuanto al valor de desplazamiento correspondiente.

\section{Conclusiones}

Los resultados obtenidos demuestran la influencia de la consideración de la IDSE en la respuesta estructural de edificaciones sometidas a carga sísmica. Esto se evidencia en el incremento que tiene lugar en los desplazamientos en el tope de la edificación y en las derivas por piso. Adicionalmente, para los casos en que se consideró la IDSE, la fluencia global de la estructura se alcanza para valores de cortante basal inferiores a aquellos en los que se consideró la base empotrada. De igual forma sucede con la aparición de las primeras articulaciones plásticas tanto en columnas como en vigas y con la generación del mecanismo de piso blando.

Por otro lado, para la relación de momento resistente columna-viga que en esta investigación ha sido empleada, las articulaciones plásticas surgen primero en las vigas y luego en las columnas, aun cuando se ha incorporado la IDSE al análisis, indicando que el mecanismo de colapso no se modifica. No obstante, el nivel de desempeño alcanzado por la edificación resulta menos adecuado al incluir la IDSE, entrando en los rangos de pre-colapso y colapso para los suelos rígido y arcilla blanda respectivamente. Esto evidencia la necesidad de incluir este fenómeno en el análisis de estructuras con las características que aquí se muestran y para los suelos estudiados. 

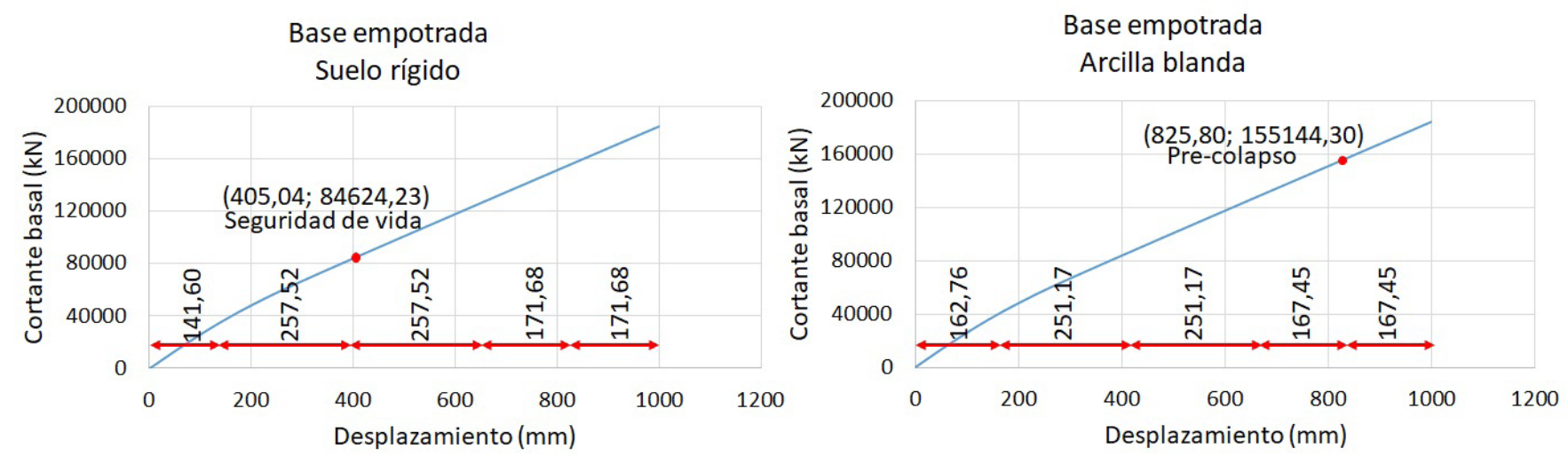

Figura 18. Sectorización de la curva de capacidad y nivel de desempeño obtenidos para cada caso analizado. Base empotrada.

Fuente: Elaboración propia.
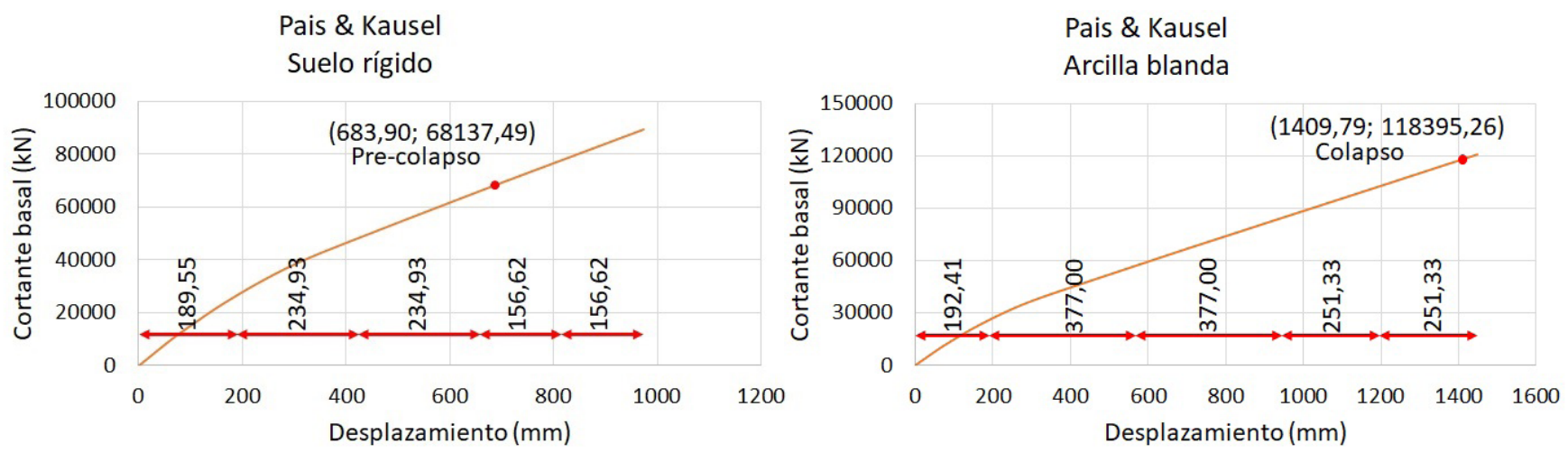

Figura 19. Sectorización de la curva de capacidad y nivel de desempeño obtenidos para cada caso analizado. Pais \& Kausel.

Fuente: Elaboración propia.

Sargsian

Suelo rígido

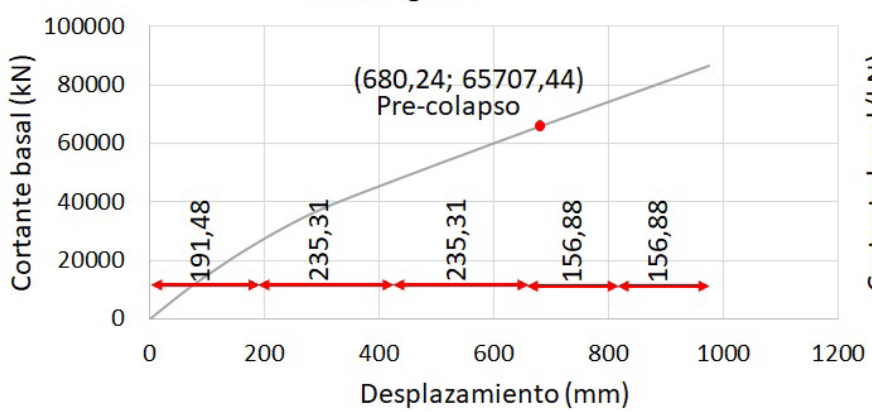

Sargsian Arcilla blanda

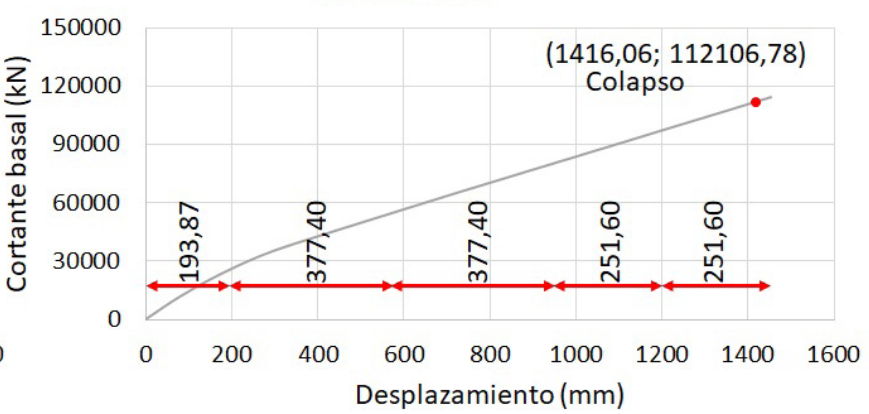

Figura 20. Sectorización de la curva de capacidad y nivel de desempeño obtenidos para cada caso analizado. Sargsian.

Fuente: Elaboración propia.

\section{Referencias bibliográficas}

[1] Oficina Nacional de Normalización, (2017) "Construcciones sismorresistentes. Requisitos básicos para el diseño y construcción. NC-46," nº 261. La Habana, Cuba, pp. 830-835.

[2] Abate, G., Massimino, M.R. and Romano, S. (2016) Finite Element Analysis of DSSI Effects for a Building of Strategic Importance in Catania(Italy), Procedia Eng., vol. 158, pp. 374-379, doi: 10.1016/j. proeng.2016.08.458.

[3] Sharma, N., Dasgupta, K. and Dey, A. (2018) A state-of-the-art review on seismic SSI studies on building structures, Innov. Infrastruct. Solut., vol. 3, nº.1, 2018, doi: 10.1007/s41062-017-0118-z.

[4] ACI 318, 2014, Building Code Requirements for Structural Concrete.

[5] IS 456, "Plain and reinforced concrete. Code of practice.," Bureau of Indian Standards,New Dehli. pp. 1-114, 2000.
[6] M. delle I. e dei Trasporti., "Norme Tecniche per le Costruzioni.," GazzettaUfficiale della Repubblica Italiana. pp. 1-198, 2018.

[7] Standards New Zealand, "Concrete structures standard," $n^{\circ}$. 1. New Zealand, pp. 1-698, 2006, [Online]. Available: papers3://publication/ uuid/84708A35-1AB5-4F68-8127-ABDAE36ECBBD.

[8] Comisión Permanente del Hormigón (2008) Instrucción de Hormigón Estructural.EHE-2008, BOE $N^{\circ}$ 203. p. 704.

[9] European Committe for Standarization, "Eurocode 8: Design of structures for earthquake resistance. Part 1: General rules, seismic actions and rules for buildings.," vol. 1, no. 2005. 2004.

[10] SEAOC, "Recommended lateral force requirements and commentary including errata." Structural Engineers Association of California (SEAOC), Sacramento, California, p. 539, 1999. 
[11] Fernández Sola, L. R., Tapia Hernández, E. and Dávalos Chávez, D. (2015) Respuesta inelástica de marcos de acero con Interacción Inercial Suelo - Estructura, Rev. Ing. Sísmica, vol. 21, nº. 92, p. 1, 2015, doi: 10.18867/ris.92.242.

[12] Fernández Sola, L. R. and León García, I. I. (2008) Diferencias en los mecanismos de colapso de edificios de acero sobre base rígida y base flexible., in XVI Congreso Nacional de Ingeniería Estructural, 2008, $\mathrm{n}^{\circ}$. 0155 .

[13] Dávalos Chávez, D., Fernández Sola, L.R. and Tapia-Hernández, E. (2013) Influencia de la Interacción Dinámica Suelo - Estructura En el comportamiento inelástico de marcos de acero., in XIX Congreso Nacional De Ingeniería Sísmica., 2013, $\mathrm{n}^{\circ}$. November.

[14] Tena Colunga, A. (2019) Soil-Structure Interaction . Reflections about its importance in the dynamic response of structures during earthquakes., Rev. Int. Ing. Estructuras, vol. 24, pp. 141-165.

[15] Villarraga, M. R. et al (2006) Estudio paramétrico de los efectos de la interacción sísmica suelo estructura.

[16] Bazán, E. and Mel, R. i, (1999) Diseño sísmico de edificios, LIMUSA Nor. México.

[17] Villarreal Castro, G. (2017) Interacción sísmica suelo-estructura en edificaciones con plateas de cimentación. Lima.

[18] Oficina Nacional de Normalización (2003) Densidad de materiales naturales, artificiales y de elementos de construcción como carga de diseño. La Habana, Cuba.

[19] Oficina Nacional de Normalización (2003) Edificaciones. Cargas de uso. La Habana, Cuba

[20] ASCE/SEI 7-10, (2013) Minimum design loads for buildings and other structures. ANSI/ASCE Standard, n . 7 98. ASCE, Reston, Virginia, pp. 1-330, 2013, doi: 10.1061/9780872629042.

[21] ACI-ASCE 352RS-02, (2010) Recomendaciones para el Diseño de Conexiones Viga-Columna en Estructuras Monolíticas de Concreto Reforzado.

[22] Fernández Sola, L.R. (2013) Influencia de la interacción dinámica suelo-estructura en la respuesta dinámica de las estructuras. Parte 1. Construcción y Tecnol. en concreto, $\mathrm{n}^{\circ}$. November, pp. 20-24.
[23] Pais, A. and Kausel, E. (1988) Approximate formulas for dynamic stiffnesses of rigid foundations. Soil Dyn. Earthq. Eng., vol. 7, n' ${ }^{\circ}$ 4, pp. 213227, doi: 10.1016/S0267-7261(88)80005-8.

[24] Bazán, E. and Meli, R. (1999) Diseño sísmico de edificios, LIMUSA NOR. México.

[25] Patel, S.K., Desai, A.N. and Patel, V. B. (2011) Effect of Number of Storeys To Natural Time Period of Building. in National Conference on Recent Trends in Engineering \& Technology, $\mathrm{n}^{\circ}$. May, pp. 13-15, [Online]. Available: http://www.bvmengineering.ac.in/misc/docs/published-20papers/ civilstruct/struct/101068.pdf.

[26] Velani, P.D. and Ramancharla, P. K. (2017) New Empirical Formula for Fundamental Period of Tall Buildings in India By Ambient Vibration Test in 16th World Conference on Earthquake Engineering, $\mathrm{n}^{\circ}$. January.

[27] Tapia Hernández, E., De Jesús Martínez, Y. and Fernández Sola, L. (2017) Demandas sísmicas en edificios regulares de acero con cimentaciones flexibles. Rev. Ing. Sismica, vol. 17, $\mathrm{n}^{\circ}$. 96, pp. 1-17, 2017, [Online]. Available: https://pdfs.semanticscholar.org/1176/ f91948d48980688a0e6506bd-40ddff91 cee6.pdf.

[28] Aguiar, R. (2007) Factor de reducción de fuerzas sísmicas en edificios de hormigón armado sin muros de corte. Barcelona, España.

[29] Vielma, J. C., Barbat, A. H. and Oller, S. (2014) Vulnerabilidad de edificios de ductilidad limitada. in Contribuciones a la evaluación de la vulnerabilidad sísmica de edificios., $\mathrm{n}^{\circ}$. September, Barcelona, España, 2014.

[30] Joy, P. V., Kuriakose, B. and Mathew, M. (2016) Pushover Analysis of Buildings Considering Soil-Structure Interaction, Appl. Mech. Mater., vol. 857, pp. 189-194, 2016, doi:10.4028/www.scientific.net/amm.857.189.

[31] Applied Technology Council (ATC), (2005) FEMA-440. Improvement of nonlinear static seismic analysis procedures. $\mathrm{n}^{\circ}$. June. Redwood City, California, p. 392, 2005, [Online]. Available: http://scholar.google.com/ scholar?hl=en\&btn $\mathrm{G}=$ Search\&q=intitle:Improvement + of + Nonlinear + Static+Seismic+Analysis+Procedures\#2\%5Cnhttp://scholar.google.com/ scholar?hl=en\&btnG=Search\&q=intitle:440,+Improvement+of+nonlinear+static+seismic+analysis+procedures\#2 